\title{
Artificial intelligence as a strategic instrument of economic development of Russia and improvement of its public administration
}

\author{
Sergey Kamolov ${ }^{1}$, Irina Molchanovskaya ${ }^{2}$, and Efim Kaunov ${ }^{1,2}$ \\ ${ }^{1}$ MGIMO University, Prospect Vernadskogo, 76, 119454 Moscow, Russia \\ ${ }^{2}$ Institute of Smart Cities Comparative Studies, Lubyanka M. Str., 16, 101000 Moscow, Russia
}

\begin{abstract}
This article gives a comprehensive study of artificial intelligence technology as an instrument of economic development of Russia and improvement of its public administration. The study considers approaches to definitions, systematizes normative legal acts regulating the activities of economic and government entities that implement AI technology across the country, provides classification of AI technologies, gives additional market analysis. The study indicates key factors impeding gradual development of AI technology and its implementation within the government regulation process. This paper provides recommendations that help mitigate the impact of the accessed impeding factors and boost $\mathrm{AI}$ integration within the priority national economy sectors in order to achieve national and global development goals.
\end{abstract}

\section{Introduction}

The level of technology development and the speed of the country's transition to the digital economy are becoming key factors that determine quality of life of the citizens, the pace of socio-economic development and the country's position on the international arena, both in the field of information technologies, as well as classic economic sectors.

The "Industry 4.0" era links technologies, dissolves boundaries between physical, biological and digital aspects of our life that becomes all the more possible due to active internet and technological expansion, as well as inclusion of artificial intelligence (AI).

The Russian President spoke on many occasions on the relevance of studying and developing AI. He emphasized that "AI, undoubtedly, is the foundation of yet another breakthrough for all mankind in its development" [1]. However, he notes that AI development brings along a number of risks which require preventive steps to be taken in order to overcome them [2].

In this regard the Russian Government faces the task of achieving the widespread use of AI "in resolving any tasks, both in the state and business segments" [3], the transition from the state of AI legacy to AI native, as well as minimizing the risks associated with the introduction of this technology.

Russia is actively taking measures aimed at the development and implementation of AI. So, in order to fulfill these tasks, the "Digital economy" national program was developed, 
and, in particular, the federal project "Artificial Intelligence". Nevertheless, there are shortcomings in the complex of measures undertaken, which have to be eliminated in order to bring about the development of AI. This will also lead to overall economic, political and social well-being. The relevance of this scientific study is determined by these circumstances.

AI became the object of research in many scientific works of Russian and foreign authors, including: Brown A. S., Shevkunov, M. A., Markotkin N., Morkhat P. M., Kholodnaya E. V., John McCarthy, Daniel Castro, Joshua New, Edward Albert Feigenbaum, Nick Bostrom.

Nevertheless, new phenomena that need to be studied in depth appear with the development of society. In this paper, attempts were made to consider them.

\section{Methodology}

The object of research is AI technology. The subject of the study is the use of AI to ensure economic development and improve public administration. The purpose of this work is to identify possible shortcomings of the system for supporting the development, implementation, and use of AI and to determine the best ways to eliminate them. To achieve these goals, the following tasks were set:

1. To study the history of the development of AI technology and to consider various approaches to the definition of AI;

2. To study the features of legal regulation of AI in the Russian Federation.

3. Identify the types of AI technologies and their applications.

4. Analyze the AI technology market in Russia.

5. Identify the main shortcomings of the support system for the development, implementation, and use of AI and suggest ways to eliminate them.

The methodological basis of the research is theoretical methods, namely, analysis, synthesis, classification, abstraction, generalization; empirical methods, namely, observation, description.

The study used normative sources: Federal Laws, Decrees of the President of the Russian Federation, Resolutions of the Government of the Russian Federation, Orders of the Government of the Russian Federation, strategic planning documents and other regulatory legal acts and standards; works of John McCarthy, Edward Albert Feigenbaum, Nick Bostrom; Morkhat P. M.; scientific articles authored by A. S. Brown, M. A. Shevkunov, N. Markotkin, E. V. Kholodnaya, etc. authors; reports from the mass media and other sources.

\section{Artificial intelligence: genesis of the technology and modern approaches to the definition of the concept}

Information and the level of its application and accessibility play a key role in the current phase of social development, also known as the "Information Society". Under such conditions, the relationship between socio-economic development and the development of digital technologies, especially "end-to-end" digital technologies (key scientific and technical areas that possess the most crucial market development impact). "End-to-end" technologies are those that simultaneously encompass several trends or industries"[4]. These technologies include:

- Neurotechnology and AI

- Virtual and augmented reality technologies

- New production technologies

- Wireless communication technologies

- Distributed registry systems 
- Quantum technologies

- Robotics and sensor components

In 2019 roadmaps of the "Digital Technologies" federal project were developed based on the "end-to-end" technologies. However, in 2020 the development of AI was separated into a standalone federal project.

What is artificial intelligence?

The history of AI technology development dates back to the 1940s. It was formed through integration of the achievements in various disciplines: Cybernetics - the science of control and communications in the animal, machine and society (N. Wiener); Information Theory a mathematical theory related to the measurement of information: the flow, volume of data, the " size" of the communication channel, etc. (K. Shenon); algorithm theory - a theory that radiates algorithm laws and the forms of their representation (A. Turing).

Along with the development of technology, the definition of AI was modified: expanded and refined. However, there is still no single, universal, comprehensively accurate definition of this concept.

Firstly, it is due to the fact that approaches to the AI concept depend on the purpose of creating a definition, its subsequent use, as well as the field of work of specialists who form the concept.

Secondly, the existence of different approaches to working out a definition of this concept stems from the existence of different approaches to the essence of technology. Thus, there are a "weak" (narrow) AI able to perform a limited range of simple tasks, and a "strong" (general) AI able to solve a wide range of tasks, which allows it to reach the intellectual level of a person [6]. Also, some authors distinguish a "superintelligence", which is able to surpass the human intellectual abilities [7].

Originally, the AI concept was proposed by John McCarthy in 1956 during the Dartmouth Conference and was described like: "AI is the science and engineering of making intelligent machines" [8]. It is worth noting that this definition does not describe the nature of the relationship between technology and an individual [9]. This was due to the fact that McCarthy believed that this area does not have a strong human connection, except for similarities in the field of performing the types of tasks inherent in living beings.

Later on, another prominent AI scientist - Edward Albert Feigenbaum - described and concretized the relationship between technology and human and gave the following definition: "AI is the part of computer science concerned with designing intelligent computer systems, that is, systems that exhibit the characteristics one associate with intelligence in human behavior - understanding language, learning, reasoning, solving problems, and so on" [10].

It must be noted that the two abovementioned approaches to AI definition have the right to exist, since AI can either be similar to human intellect, or completely differ from it.

It is noteworthy to consider the approach this term is defined in the documents of the strategic planning of the Russian Federation. For instance, the National Strategy contains the following definition: "AI is a set of technological solutions that can simulate human cognitive functions (including self-learning and finding solutions without a predetermined algorithm) and obtaining results comparable, at the very least, to the results of human intellectual activity when performing specific tasks" [12]. While analyzing this definition, we can conclude that the Russian Federation proceeds from the fact that AI technology is strongly connected with humans: it simulates cognitive architecture of human intelligence, and also considers technology from the point of view of the "strong" AI.

Due to complexity of this term, it seems necessary to interpret it in an comprehensively way. In this regard, according to the authors, the most exhaustive definition was given by $\mathrm{P}$. Morkhat. Taking into account and generalizing various approaches, $\mathrm{P}$. Morkhat proposed the following definition: 
"AI is a fully or partially autonomous self-organizing computer-software virtual or cyberphysical (also bio-cybernetic), system (unit) with the following preset capabilities:

- anthropomorphic-cognitive thinking and actions, such as image recognition, symbolic systems and languages, reflection, reasoning, modeling, imaginative (meaninggenerating and sense-perceiving) thinking, analysis and evaluation;

- $\quad$ self-reference, self-regulation, self-adaptation to changing conditions, self-restraint;

- self-maintenance in homeostasis;

- genetic algorythm (a heuristic search algorithm that preserves important aspects of "parental information" for "subsequent generations" of information), accumulation of information and experience;

- learning and self-learning (based on their own mistakes and experience); selfdevelopment and self-application of self-homologation algorithms;

- independent development of tests for their own testing, independent self-testing and testing of computer and, if possible, physical reality;

- anthropomorphic-intelligent independent (including creative) decision-making and problem-solving."[13]. This definition highlights various approaches to the essence of the technology, as well as reflects a wide range of AI capabilities.

It should also be noted that in 2021 the "Artificial intelligence. Concepts and terminology" ISO/IEC terminology standard is planned to be translated into Russian. This may allow us to create a single definition of the term for all market participants. This step will contribute to improving the coordination of actors developing and using this technology.

$\mathrm{AI}$ is one of the most dynamically developed technologies that contribute to increasing the pace of economic growth, improving public governance and well-being. The study of this phenomenon is reflected in the works of many researchers. It seems that the definition presented by P. M. Morkhat gives the most comprehensive explanation.

\section{Special aspects of ai legal regulations in Russia}

$\mathrm{AI}$ is one of the most rapidly developing technologies, the advancement regulation of which is impossible without defining its basic principles. One of the most significant tools in this area is the self-regulation tool. An example of this tool is the Asilomar Principles adopted in 2017. Although some do not possess legal force, they influence behavior in this area, determine the vector of AI development and direct scientific research. These principles have received quite a broad support from the scientific community: more than 3,500 scientists, experts, and entrepreneurs have signed these principles. They cover 3 main areas:

- Research Issues: special attention is paid to establishing a constructive dialogue between policy makers, scientists, developers and researchers.

- Ethics and Values: dedicated to creating an open, secure system for the development, implementation and use of AI.

- Long-term Issues: they consider aspects related to risk assessment and opportunities of AI [14].

It is worth noting that to date a single AI regulatory system has not yet been formed. This is due to a number of factors: there is no unified approach to determining the subject of AI regulation; there is no effective mechanism for delegating decision-making to AI systems; there is no system of responsibility for illegal AI behavior; there is no transparency of the algorithms according to which an AI system works [15].

Nevertheless, Russia has already started developing a regulatory system. It is based on:

- The Constitution of the Russian Federation;

- $\quad$ Federal Law No. 172 «On Strategic Planning of the Russian Federation»; 
- $\quad$ Decrees of the President of the Russian Federation No. 204 "On National Goals and Strategic Objectives for the Development of the Russian Federation for the Period up to 2024", No. 203 "On the Strategy for the Development of the Information Society in the Russian Federation for 2017-2030", No. 642 "On the Strategy for Scientific and Technological Development of the Russian Federation" and etc;

A number of strategic planning documents defining the main vectors of AI technology development were also created:

- $\quad$ National Strategy for AI Development for the period up to 2030 (hereinafter - the National Strategy);

- $\quad$ Strategy for Information Society Development in the Russian Federation for 20172030

Additionally, in order to create the foundation for legal regulation of new public relations arising in connection with the creation and implementation of AI systems, as well as to minimize the impact of factors that hinder the qualitative development of technology, the Concept of Regulating AI and Robotics Technology up to 2023 was created.

The key instrument for implementing the National Strategy is the "Artificial Intelligence" federal project. Its goal is to create favorable conditions for scientific research, software creation and development, demand stimulation, and AI technologies popularization [16].

Additionally, according to the Decree of the Government of the Russian Federation No. 1750 , AI was included in the list of technologies gradually integrated in the framework of experimental legal regimes. This step will help developing technologies that are not yet fully regulated by the current legislation. This legal regime is already in effect in Moscow starting July 1,2020 . The coordination council is responsible for strategy and mechanisms designed to implement these so-called "digital sandboxes", which at the conclusion of the experiment must submit a statement on the feasibility or inexpediency of making changes to the existing legal regime [17].

The goal of creating such a regulatory system is to provide favorable conditions for the creation, implementation and use of $\mathrm{AI}$ in order to increase the pace of economic development, improve the quality of life of citizens, and strengthen the country's position on international markets.

Therefore, the momentum of technology development makes it necessary to regulate the development, implementation, and use of AI. Currently there are Federal Laws, Presidential Decrees, and Strategic Development Documents that regulate this area, but there is still no single, comprehensive and effective system for AI regulation.

\section{Al technologies classification}

AI undergoes rapid development, gradually embracing all aspects of our lives. For a more indepth take on how AI will change our way of life, let us take a closer look at AI technologies: what is their core essence, where they are being used, and how will they affect socioeconomic development and public regulation.

The Moscow Industrial Development Agency offers the following classification of AI technologies: 


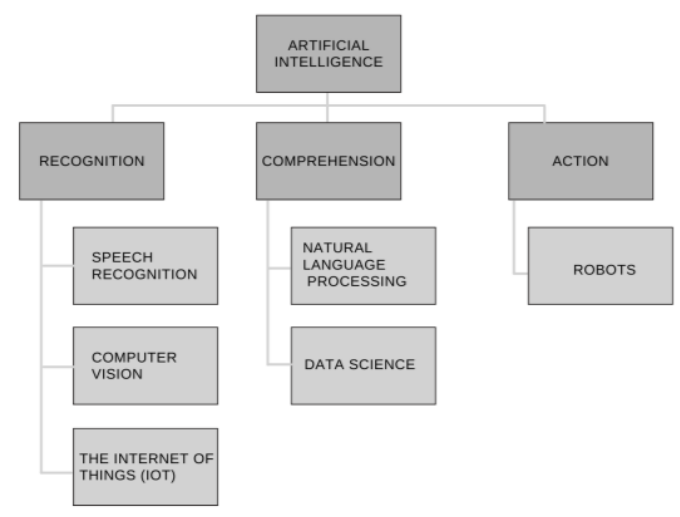

Fig. 1. Classification of AI technologies [18]

It must be emphasized that these technologies are closely related to each other: progress in the development of one of the technologies contributes to acceleration in the development of another. Moreover, when implementing AI technologies in various areas, as a rule, they are not limited to introduction of only one technology, on the contrary, they rather introduce a whole set of AI technologies in order to achieve great results.

Let us study AI technologies in more detail.

\subsection{Recognition:}

1. Speech recognition - a technology that allows you to convert speech into text or other type of digital information.

This technology is actively used in call centers of various organizations, which allows customers to receive services more quickly. Speech recognition technology is also used in various online translation services [19].

2. Computer vision - a technology that allows you to process visual information in order to obtain useful knowledge.

The key objectives of the technology are:

- Object detection - determines the location of objects within the coordinate system.

- Object tracking - a logical extension of the object detection technology, which allows you to track the location of objects in motion and predict their further movement.

- Image segmentation - a technology that allows one to determine which parts of the image relate to a particular object.

- Depth and Distance Estimation - a technology that estimates the distance to objects in photos and videos.

This technology is actively used in the digital economy, in agriculture, in healthcare, in self-driving cars, unmanned aerial vehicles [20].

3. The Internet of Things (IoT) - a technology that allows you to create a system in which data can be transmitted between devices. It allows you to collect, process and transfer information between the devices within a system [21].

\subsection{Comprehension:}

1. Natural language processing — this technology helps computers understand, interpret, and process speech. The technology is based on linguistics and computer technologies [22].

It allows you to: 
* organize information

* sentiment analysis

* summarize information

* perform machine translation [23].

This technology is used to analyze posts in social networks, news and scientific articles

2. Data science - a technology based on statistical methods and algorithms that allow you to obtain knowledge from the results of observations and later use this data for decisionmaking in science, business, and public regulation.

This technology allows you to:

* Recognize video, text, speech

* Build recommendation models

* Segment information [24].

\subsection{Action:}

1. A robot - an executive mechanism programmed for at least two levels of mobility that has a certain degree of autonomy and is also able to move in order to perform its tasks.

There are two classes of robots:

- Industrial robots used for industrial automation purposes

- Server robots, which specialize in servicing equipment, as well as performing tasks that are necessary for humans [25].

It should be noted that the National Strategy defines a slightly different approach. Thus, within the framework of this strategic planning document AI technologies include technologies based on the use of artificial intelligence (See Figure 2).

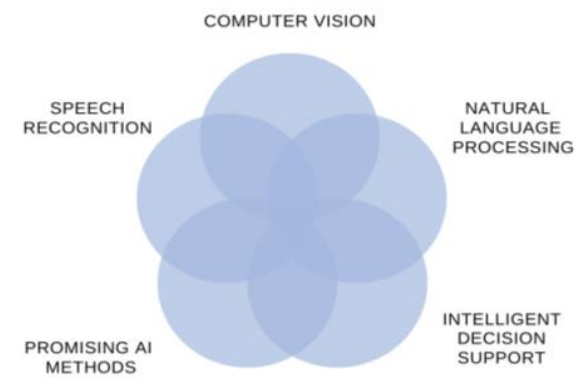

Fig. 2. Classification of AI technologies Source: complied by the authors on the basis of the Decree of the President of the Russian Federation of 10.10.2019 N 490

These approaches overlap in many areas. However, the National Strategy separately specifies the following technologies:

Intelligent Decision Support - an automated computer system the activities of which are aimed at helping decision-makers in difficult conditions, that is AI-based in performing tasks. This technology allows for a complete and objective analysis of the problem, as well as to make an estimation of a situation's development [26].

Promising AI methods - a collective term that includes technologies intended to create fundamentally new scientific and technical products, including «strong» AI development. For instance, they include automatic machine learning, autonomous solution of various problems, algorithms for resolving problems based on data with partial markup and (or) insignificant amounts of data, and etc [27]. 
Additionally, within the framework of the National Strategy robotics and unmanned vehicle management are regarded as related areas and not as AI technologies.

Therefore, classification of AI technologies fully covered in the report submitted by the Moscow Industrial Development Agency with the support of the Department of Investment and Industrial Policy of Moscow. This classification covers three main areas of AI: recognition, comprehension and action, and classifies technologies within these areas.

\section{Analysis of the ai technologies market}

AI is a technology that serves as a key driver of the new technological and industrial revolution. According to the results of the Digital Leader study with the support of PwC, IDC and CROC, AI and machine learning, the IoT and robotization of processes will lead to the greatest amount of changes in Russian companies [28]. These trends are also supported by data on the growth of investment in AI in the world. In 2020 this figure increased by $40 \%$ and reached $\$ 67.9$ billion. Investors were particularly interested in companies that use AI in the development of new drugs, specialize in molecular medicine, develop self-driving cars, and create educational AI products [29].

The lead countries in the development, study, implementation and use of AI are traditionally the U.S. and China.

The U.S. is a leader in the AI technology development level. This is due to the fact that the U.S. National AI Development Strategy has a well-devised system of government support for AI initiatives. It is important to note that it is not only formally legislated, but also effectively functions. The U.S. annually plans to increase spending on non-defense AI, so in 2021 it will spend $\$ 1.5$ billion, and in 2025 this figure will amount to $\$ 8$ billion [30]. The U.S. also successfully manages to attract the best foreign specialists.

China currently ranks second in terms of AI development. As part of the strategic state program for the AI development until 2030 China should become the leader in this area. For China AI development is one of the most important domestic political tasks, since the work of the social rating system is AI based, aimed at ensuring socio-political stability. Additionally, AI for China is a tool for making a qualitative leap in the development of the military-technological sphere. The main driver of China's success in this area is the massive amount of data generated by Chinese residents. China also actively promotes international scientific and technological exchanges and pays special attention to personnel training.

As far as Russia is concerned, the county «possesses a significant potential in order to become one of the international leaders in development and implementation of AI technologies» [31], however, it is unlikely that Russia will be able to achieve this goal in the nearest future [32].

It is worth mentioning that it is difficult to determine Russia's position in the technology race among other countries. This is due to the fact that different estimation methods were used in different ratings. Nevertheless, considering Russia's position in a number of ratings will allow us to put together a more objective picture. So, let's look at the main ratings:

1. Government AI Readiness Index 2020 by Oxford Insights and IDRC. Russia is ranked 33rd between the Czech Republic (32nd place) and Slovakia (34th place). The U.S. took the first spot. All indicators of this rating are divided into three groups:

- Government. Within this indicator it is noted that Russia recognizes that AI serves as the main strategic advantage, and it also seeks to improve its position in this area. Experts note that AI technologies are more widely used in the military, rather than the civilian sphere. There are also a number of concerns that Russia may use AI technologies in an irresponsible and unethical manner towards its citizens. 
- Technology sector. AI development in Russia is centralized: the main driver of AI development in Russia is the government, not the private sector. Experts also emphasize that Russia needs to improve its position in the private technology sector. The country's strong point is STEM education.

- Data and infrastructure. Russia has fairly high-speed Internet and cellphone coverage rates, but there is a digital division between different territories of the country. Russia is also characterized by a high level of state control over the Internet [33].

2. The Global AI Index from Tortoise Media. Russia ranks 31 st in the ranking, between Italy (30th) and Malta (32nd). This rating covers three main aspects: AI implementation, innovation, and investment. Russia received the lowest rating in working environment (implementation), as well as commercial development (investment).

Additionally, in order to create a more objective assessment of the degree of AI development it is necessary to consider other key indicators in this area:

1. The number of supercomputers that rank in the top 500 most powerful in the world. Now there are only two such supercomputers in Russia. China (217 supercomputers), the United States (116 supercomputers) and Japan (43 supercomputers) are among the leaders in this area [34].

2. The Number of scientific publications. Russia ranked 16th in 2019, 25th from 1996 to 2019 [35].

3. Computer science education. 22 Russian universities are among the top 800 best universities in this field. For comparison, this list includes 123 universities from the U.S. and 82 from China. The highest rankings in this rating among Russian universities were received by MIPT (91st place), ITMO (101-125 place) and MSU (126-160 place) [36].

4. The number of AI startups. Currently there are 217 startups in Russia. The most promising ones include N-Tech Labs (develops facial recognition technology), Wallarm (deals with web security technologies to protect online businesses from hacker attacks), Bbrain (a blockchain platform for creating AI applications) and others. In the U.S. there are9376 startups, in China - 1392 [37].

Therefore, Russia holds a huge potential in AI technology development. Currently we have already achieved good results in the field of education related to computer technologies, the level of Internet coverage, and the availability of data for the population. Nevertheless, Russia is still not a leader in this field.

\section{Factors impeding ai development in Russia, potential steps for their mitigation}

In Russia, socio-economic development has long been a discussion topic. However, in the current crisis conditions it is of particular interest. 2020-2021 were years of serious unrest: the COVID-2019 pandemic, unstable oil prices, introduction of new economic sanctions - all these factors changed the way of life not only for individual citizens, but for the country as a whole.

These factors have led to a drop in Russian revenues, and the budget deficit is expected to be $2.4 \%$ of GDP in 2021 [38]. In conditions of limited resources it is especially important to manage them effectively, so there is a need to improve the mechanism of socio-economic development and public administration. This brings us to the question whether AI can become one of the sources of overcoming the current crisis?

The use of AI technologies allows one to: automate routine processes and dangerous types of work, free employees from monotonous work and promote optimal decision-making.

These facts contribute to a more efficient use of resources, including human potential. 
However, it should be noted that there are certain circumstances that stand in the way of maximizing the benefits of using $\mathrm{AI}$ as a tool for economic development of the country and improving its public regulation. Let's consider the main ones:

\section{Imperfect legal regulations.}

AI technologies are one of the fastest growing areas and many technological solutions are significantly ahead of the development of the regulatory framework. This fact is not only due to the specifics of these technologies, but also to untimely legal regulation. A striking example of this is the delay in adopting the AI Development Strategy. The document was adopted only in 2019. It seems that the solution to this problem may be a more active use of "regulatory sandboxes". This measure will allow one to test the technology in a certain area and only then it will allow one to make informed decisions about the regulation of technology throughout the country more quickly.

"Soft" forms of regulation can also be an effective tool for maximizing the benefits of AI technologies. The government should support and encourage adoption and implementation of documents, codes of ethics and other instruments of voluntary application. It seems that the creation of self-regulation tools (such as the Asilomar Principles) will contribute to the development of self-awareness among developers of AI technologies and those who put them to use. Additionally, these tools will contribute to greater support for the development of AI within society, as this will ensure that these technologies will not be used against it.

\section{Insufficient development of international cooperation.}

The development of international cooperation in AI, exchange of technologies and specialists will allow us to achieve greater progress in AI development.

One of the main tools for the development of international cooperation is Russia's participation in various conferences, development of international standards, and negotiations held at key international venues: the OECD, ITU, IEC, and ISO.

\section{Unfavorable investment climate.}

The next promising direction of accelerating AI development in Russia is to improve the investment climate and create a favorable business environment.

The pressing need to improve the mechanism for attracting investment in the Russian economy stems from the necessity not only to create new technologies and develop innovative products that are competitive on the world market, but also to increase the share of non-resource non-energy exports, create new high-tech jobs and improve the skills of employees working for high-tech enterprises. To implement the objectives of the investment policy, the Government of the Russian Federation adopted the "Action Plan to Accelerate the Growth rate of Investments in Fixed Assets and Increase their Share in the GDP up to 25 percent". However, the adoption of these measures is insufficient, as evidenced by the results of the Ernst \& Young survey, which was held within 62 foreign companies [39].

According to this study, one of the main obstacles to our business is frequent legislation changes. There is no doubt that the legislation should be changed to meet the challenges of the time, especially in such a rapidly developing area, but 50 percent of respondents said that the changes occur more often than necessary. Moreover, 34 percent of respondents are concerned about the discrepancy between the law and the current business environment. Additionally, the high level of administrative barriers creates difficulties for doing business for 39 percent of the survey participants.

It seems that reducing the impact of such negative factors as unjustifiably frequent changes in legislation, a high level of administrative barriers and discrepancies between the law and the current business environment will create a more favorable business environment and make the Russian Federation more attractive for investment.

\section{High rate of intellectual migration from Russia and lack of qualified personnel}

Special attention should be paid to the high rate of intellectual migration from Russia. According to Rosstat, 77.3 thousand Russians left the country in 2018. However, it is worth 
noting that this migration statistics does not reflect the real picture, since it only accounts those who, when moving from Russia, underwent the legal procedure within the migration register. Yulia Florinskaya, a leading researcher at the Institute for Social Analysis and Asessment of the Russian Academy of Sciences, believes that 100-120 thousand people move annually to the countries of "classical" immigration alone. It is important to note that young, ambitious people who are ready to work are migrating from the country. Such people can become a source for AI development of the country.

In this regard it is necessary to further improve the grant system of education in universities, expand cooperation with foreign universities, establish a student exchange system with their subsequent return.

\section{The problem of AI legal regulation in government and municipal administration.}

The solution of all the above-mentioned tasks requires effective public administration. AI can become a tool for reducing the number of routine operations, reducing costs and increasing decision-making speed.

AI technologies should be implemented gradually. The first step should be the use of AI in operations that do not depend on critical decisions. In the future, these technologies can be used for processing citizens' appeals, providing of public services, and implementing control and supervisory measures.

Currently it is possible to stimulate the introduction of AI in public administration by creating regulatory opportunities for delegating tasks to AI technologies, forming a mechanism for assessing AI compliance with security requirements and evaluating their effectiveness, as well as compliance with human and civil rights and freedoms.

In conclusion it is important to note that AI development will make a much-needed "economic leap" and improve the quality of public services. However, it is also necessary to keep in mind the circumstances that can lead to a decrease in the pace of AI development, namely, the imperfection of regulatory legal regulation, insufficient development of international cooperation, unfavorable investment climate, high rate of intellectual migration from Russia and lack of qualified personnel, the problem of legal regulation of the use of technologies AI in government and municipal administration. Overcoming these obstacles, maximizing the positive impact of AI development, and implementing these technologies in public administration should be among public administration priority goals.

\section{Conclusion}

Thus, on the basis of the study, the following conclusions can be drawn:

1. AI technologies are among the most dynamically developing technologies, and they are already actively changing our lives, penetrating into all spheres of life. There is a significant amount of scientific research on this topic, in which various approaches of defining AI have been presented. And according to the authors, the most comprehensive approach was presented by P. M. Morkhat.

2. At the moment, the development, implementation and use of AI is regulated by Federal Laws, Presidential Decrees, and Strategic Development Documents. However, the system that they form at the moment cannot be considered as exhaustive and comprehensive.

3. The classification of AI technologies is most fully reflected in the report submitted by the Moscow Industrial Development Agency. This classification includes three main areas of AI activity: recognition, comprehension, and action, and classifies technologies within these areas.

4. Russia has a huge potential in the development of AI technologies. At the moment, we have already achieved good results in the field of education related to computer technologies, the level of Internet penetration, and the availability of data for the population. However, in 
Russia, AI is developing centrally: the main engine of AI development in Russia is the state, not the private sector. Russia is characterized by a high level of state control over the Internet. Also, a significant part of the development in the field of AI is related to the military, not the civilian sphere. And there is a significant digital divide between different territories in the country. These circumstances show that Russia still can not be considered a leader in this area.

5. In order to strengthen Russia's position in the international arena in this area, it is necessary to solve a number of problems. Among them are:

- Imperfect legal regulation. The solution to this problem can be a more active use of "regulatory sandboxes", as well as the use of "soft" forms of regulation, such as supporting and encouraging the adoption and application of documents, codes of ethics and other voluntary application tools.

- Insufficient development of international cooperation. In this regard, it is necessary to take a more active part in various conferences, the development of international standards, negotiations held at key international venues: OECD, ITU, IEC, ISO, etc.

- Unfavorable investment climate. Reducing the impact of such negative factors as unjustifiably frequent changes in legislation, high administrative barriers and discrepancies between the law and the current business environment will create a more favorable business environment and make Russia more attractive for investment.

- High rates of intellectual migration from Russia and a lack of qualified personnel. In this connection, it is necessary to further improve the system of educational grants in universities, expand cooperation with foreign universities, establish a system of exchange of students, with their subsequent return.

- The problem of legal regulation of the use of AI technologies in state and municipal administration. To overcome this problem, it is necessary to create regulatory opportunities for delegating tasks to AI technologies, forming a mechanism for assessing the compliance of AI systems with security requirements and evaluating their effectiveness, as well as compliance with human and civil rights and freedoms.

Thus, the development of AI will allow us to make a much-needed "economic leap" and improve the quality of public services. Overcoming the above-mentioned obstacles and maximizing the positive effects of the development of AI, the introduction of these technologies in public administration should be one of the priorities of public administration.

\section{References}

1. TASS Agency, Putin called artificial intelligence the basis for a new breakthrough in the development of mankind (2020)

2. Interfax, Putin urged to stop all risks associated with AI in advance (2020)

3. Ministry of economic development of Russia, Deputy Minister Oksana Tarasenko in an interview with CNews - about the development of artificial intelligence in Russia (2021)

4. Order of the Ministry of Communications of the Russian Federation of 01.08.2018 №428

5. Presidium of the Government Commission on Digital Development, the Use of Information Technologies to Improve the Quality of Life and Business Conditions, Passport of the federal project " Digital Technologies" (Protocol № 9 of 28.05.2019)

6. D. Castro, J. New, The Promise of Artificial Intelligence, 3 (2016)

7. E.V. Kholodnaya, Bulletin of the O. E. Kutafin University, 12, 94 (2019)

8. J. McCarthy, Mechanisation of Thought Processes, Proceedings of the Symposium of the National Physics Laboratory, 77 (1959) 
9. J. McCarthy, book review of B. P. Bloomfield, The Question of Artificial Intelligence: Philosophical and Sociological Perspectives, in Annals of the History of Computing 10, 3 (1998)

10. Helferich, The Handbook of Artificial Intelligence, 123 (1984)

11. N. Bostrom, Artificial intelligence: Stages. Threats. Strategies: Per. s engl., 61 (2016)

12. Decree of the President of the Russian Federation of 10.10.2019 N 490

13. P. M. Morkhat, Artificial intelligence: a legal view, 69 (2017)

14. Principles of working with AI, developed at the Asilomar conference (2017)

15. Order of the Government of the Russian Federation of 19.08.2020 N 2129-r

16. Passport of the federal project "Artificial Intelligence" of the national program "Digital Economy of the Russian Federation"

17. Resolution of the Government of the Russian Federation of 28.10.2020 N 1750

18. Artificial intelligence technologies

19. Natural Language Understanding / Processing Speech / Voice recognition

20. Sberbank Robotics Laboratory, Analytical review of the global robotics market (2019)

21. What is the Internet of Things Internet of Things, IoT

22. SAS, Natural Language Processing (NLP) What it is and why it matters

23. Best Practices Report, Advanced Analytics: Moving Toward AI, Machine Learning, and Natural Language Processing By Fern Halper

24. Data Science

25. GOST R 60.0.0.4-2019 / ISO 8373: 2012 Robots and robotic devices. Terms and definitions (2019)

26. A. S. Buryy, M. A.Shevkunov, Information and economic aspects of standardization and technical regulation: Scientific Online Journal, 5(27) (2015)

27. Decree of the President of the Russian Federation of 10.10.2019 N 490

28. Trends and technologies 2030

29. Stanford University, The AI Index Report Measuring Trends In Artificial Intelligence

30. National Security Commission on Articial Intelligence, Final Report National Security Commission on Articial Intelligence

31. Decree of the President of the Russian Federation of 10.10.2019 N 490

32. N. Markotkin, E. Chernenko, Development of artificial intelligence technologies in Russia: goals and reality (2020)

33. Oxford Insights, Government AI Readiness Index 2020

34. TOP500 LIST (2020)

35. Scimago Journal \& Country Rank

36. The World University Rankings, World University Rankings 2021 by subject: computer science (2021)

37. Artificial Intelligence Startups in Russia

38. TASS Agency, The federal budget deficit of Russia in 2021 will amount to 2.75 trillion rubles

39. Ernst \& Young, FIAC survey: foreign business in Russia (2020) 